\title{
Internal and external cue use following septal ablation in the rat
}

\author{
KENNETH E. KRATZ and JAMES C. MITCHELL \\ Kansas State University, Manhattan, Kansas 66506
}

\begin{abstract}
As a result of a proposed response-produced cue deficit in rats with septal damage, an attempt was made to determine whether rats with such damage would be overly dependent on exteroceptive cues. Normal and septal lesioned rats were trained to perform a behavioral chain where both exteroceptive and response-produced cues could be used. During extinction, these two sets of cues were placed in conflict in order to determine their relative contributions to performance of the chain. Septal rats primarily used response-produced cues, whereas normal animals randomly used the available cues. It was concluded that normal and septal lesioned rats differed in their use of external and internal information, but in a direction opposite to that suggested by the literature.
\end{abstract}

Ellen and Butter (1969) have demonstrated that the addition of a cue light significantly improves performance of rats with septal lesions on a task requiring low rates of responding (DRL). Since the cue light signals the end of the required delay of responding, they conclude that the septal deficit on DRL schedules is the result of an inability of these animals to use the response-produced, proprioceptive stimuli used by normal animals. We have demonstrated in our own laboratory (Mitchell \& Kratz, Note 1) that, unlike normal rats, septal-lesioned rats which are deficient in two-bar ratio performance (Ellen \& Kelnhofer, 1971) are facilitated by the addition of external cue lights. Srebro's (1974) study of retention deficits in successive position reversals indicates that the addition of a visual cue also improves performance of rats with septal lesions on this task. Srebro further shows that the saliency of the visual cue with respect to its correlation with the position reversal will systematically improve performance on the task as saliency increases. Thomas (1972), in a thorough investigation of spontaneous alternation, was able to determine that rats with septal lesions consistently choose to respond to external sensory rather than internal somatomotor cues. Each of these studies dẹmonstrates the septal-damaged animal's increased dependency on external information and support Ellen and Butter's hypothesis. In agreement with this hypothesis, reviews by Lubar and Numan (1973) and Caplan (1973) emphasize the deficiency of animals with septal lesions in their use of response-

This research was supported in part by a National Institute of Health training grant, MH 08359, and by a Biomedical Sciences support grant, RR 07036. Kenneth E. Kratz is now at the University of Virginia Medical School, Department of Physiology. Requests for reprints should be sent to James C. Mitchell, Psychology Department, Kansas State University, Manhattan, Kansas 66506. produced cues. A number of studies, however, indicate that rather than being deficient in their use of internal information, animals with septal area lesions are quite efficient in their use of these types of cues. Van Hoesen, MacDougall, and Mitchell (1972) have shown that animals with septal lesions are more efficient than normal animals on a two-bar ratio schedule where a high response output is required. Morgan and Mitchell (1969) and Sodetz (1970) have shown that rats with septal lesions are superior to normal animals when performing on a Sidman avoidance schedule. Since performance on these tasks presumably requires the use of responseproduced cues, such findings are inconsistent with the notion that septal damage leads to a deficit in the use of response-produced cues. Although making it difficult to accept Ellen and Butter's (1969) responseproduced cue deficit hypothesis, such inconsistency does allow for the notion that the septal lesion does alter in some way the use of internal and/or external information. The purpose of the present study was to further examine the use of such cues by normal animals and animals with septal lesions.

Thus, normal and septal lesioned rats were trained to perform a behavioral chain where both exteroceptive and response-produced cues could be used. During extinction testing, the exteroceptive cues were placed in conflict with the previously conditioned response-produced cues and thereby provided a means to determine the relative contribution to performance of each of these sets of cues.

\section{METHOD}

\section{Subjects}

The subjects were 20 experimentally naive, male, Long-Evans hooded rats, approximately 150 days of age and weighing 200 to $300 \mathrm{~g}$. All rats were maintained on ad-lib laboratory chow and a 23-h water-deprivation schedule. 


\section{Surgery and Histology}

All operations were conducted under Equi-Thesin anesthesia. Septal lesions were made electrolytically by passing $1.5-\mathrm{mA}$ anodal current for $20 \mathrm{sec}$ through a 30-ga stainless steel electrode, insulated except for a $0.5-\mathrm{mm}$ bare tip. The circuit was completed with a rectal cathode. Coordinates were measured in millimeters from bregma, midline, and top of the skull, with the head at a $5^{\circ}$ angle to the horizontal plane (nose up). Bilateral electrode insertions were made at $1.7 \mathrm{~A}, 0.5 \mathrm{~L}$, and $6.0 \mathrm{D}$. An intramuscular injection of 30,000 units of Flocillin was administered postoperatively.

As a control for incidental damage to the auditory system occurring as a result of ear-bar insertion, animals of the normal control group were also placed in the stereotaxic apparatus. No further surgical treatment was given, however.

Following behavioral testing, all rats were anesthetized and perfused intracardially with $0.9 \%$ saline and $10 \%$ Formalin. After extraction, all brains were fixed in Formalin, and the brains of lesioned animals were dehydrated in pyridine, and embedded in celloidin. Coronal sections 20 microns thick and 60 microns apart were cut and stained with cresyl violet.

\section{Apparatus}

A circular operant chamber designed to minimize spatial cues was used. The chamber was $58.5 \mathrm{~cm}$ in diameter and had three levers placed equidistant around the circumference of the chamber with a cue light placed above each lever. Water reinforcement was presented in a small depression tooled into a circular piece of brass, located in the center of the floor. Diffuse, indirect illumination was provided by four $25-\mathrm{W}$ bulbs. The test chamber was located in a sound-attenuating chamber built of 2-cm plywood lined with acoustical tile. A blower provided ventilation and background noise within the sound-attenuating chamber. Each bar response was recorded by an event marker and sequentially recorded on paper tape for subsequent computer analysis.

\section{Procedure}

Subjects were divided into four groups of five animals each. Two of the groups were given bilateral lesions of the septal area. One normal and one septal group were trained to a criterion of 250 reinforcements on a chained behavior in which the animals were required to follow the cue lights above the three levers in a right-going sequence for two rotations around the chamber before receiving reinforcement. The other normal and septal groups were trained to a criterion of 500 reinforcements on the same task. This second criterion level was included to ensure that one group of animals would be tested at an asymptotic level of performance on the chained behavior.

Shaping and discrimination training. During initial shaping and discrimination training, a V-shaped metal divider was placed in the chamber to limit the training space to one lever and the reinforcement cup. Animals were shaped to barpress in the presence of a cue light over the lever. After 150 barpresses on a continuous reinforcement schedule, the animal was brought under stimulus control of the cue light. The cue light was presented only after the animal had refrained from making a leverpress for $20 \mathrm{sec}$. The first leverpress, after the cue light came on, resulted in reinforcement and also turned the cue light off.

Following 150 reinforcements on this schedule, the divider was removed and the cue light was lit over one of the three levers. A barpress at this lever resulted in reinforcement, with the cue light being lit at random over one of the three levers. If an incorrect response was made, reinforcement was not delivered and the cue light remained on over the correct lever.

Chained behavior training. Training of the chained behavior was then begun, and consisted of three phases. First, the animals were given 50 reinforcements for performing a chain in which they were required to press Lever $1\left(R_{1}\right)$ when the cue light over that lever was lit $\left(S_{1}\right)$. The light over Lever 2 was then lit $\left(S_{2}\right)$, and the animal was required to press Lever $2\left(\mathbf{R}_{2}\right)$. The cue above Lever 3 then came on $\left(\mathrm{S}_{3}\right)$, and the animal was required to press Lever $3\left(\mathbf{R}_{3}\right)$, at which time water $\left(S^{R}\right)$ was delivered and the sequence was started again $\left(S_{1}-R_{1}-S_{2}-R_{2}-S_{3}-R_{3}-S R\right)$. Incorrect responses were not reinforced, nor did they change the position of the cue light. During this phase of the training, the cue lights came on immediately following the previous response in the chain.

The second phase of this training was similar to the first phase, except that a 2-sec delay was instituted between the leverpress and lighting of the next cue light. Barpresses on the correct lever during the 2 -sec delay resulted in reinforcement. Phase 2 continued for 50 reinforcements.

At this time, a third phase began in which the animals were required to perform two complete sequences of the right-going response $\left(S_{1}-R_{1}-S_{2}-R_{2}-S_{3}-R_{3}-S_{1}-R_{1}-S_{2}-R_{2}-S_{3}-R_{3}-S^{R}\right)$. Daily 30-min sessions were run on this schedule until the animals reached their specified criterion levels. The chained behavior could be maintained through the use of two sets of cues; the cue lights or external cues and/or the internal cues produced by the animal's rightgoing movements. During this portion of the experiment, internal and external cues were redundant with respect to the information they provided.

As an added control for differential spatial cues, in addition to those provided by the external cue lights or internal cues generated by the animal's behavior, the starting position in the chamber was changed at the beginning of each day of training.

Extinction testing. A test session of $30 \mathrm{~min}$ was run on the day following achievement of the criterion level. This test session was conducted in extinction with the cue lights following a left-going direction, opposite in direction of rotation to that of the acquisition training. If the cue light over Lever $1\left(R_{1}\right)$ was lit $\left(S_{1}\right)$, a press of this lever resulted in the illumination of the cue light $\left(S_{3}\right)$ over Lever $3\left(R_{3}\right)$ and the switching off of $S_{1}$. Pressing Lever $3\left(R_{3}\right)$ then resulted in the illumination of $S_{2}$, pressing Lever $2\left(R_{2}\right)$ resulted in the illumination of $S_{1}$. This meant that during extinction testing, the pattern of the external cue lights was in conflict with the pattern of the previously reinforced internal responseproduced cue information.

\section{RESULTS}

\section{Histological}

All lesions were examined to determine the extent of damage to the septal nuclei and the amount of damage to neighboring structures. Septal lesions began in the vicinity of the genu of the corpus callosum and extended caudally to the descending columns of the fornix. Dorsally and ventrally, the lesions were confined to the area between the corpus callosum and the anterior commissure. Laterally, lesions were confined to the area between the lateral ventricles. Every lesion destroyed the major portions of the lateral and medial septal nuclei except in a few instances where the extreme ventromedial aspect of the septum was spared. Incidental damage to the corpus callosum, cingulate cortex, caudate, anterior commissure, and columns of the fornix was minimal or nonexistent in all lesions. No differences were observed in the two groups of lesioned animals with respect to amount or location of tissue damage.

Data from one animal in each lesioned group was not included in any of the analyses as a result of histological examination of the brains. 


\section{Data Conversion}

Responses were recorded sequentially on punched paper tape during the last 30 min of acquisition training and during the extinction session. The extinction session was analyzed by dividing it in half on the basis of number of responses in the session. This provided two segments of testing during the extinction phase of the experiment.

A computer analysis provided a tabulation of overlapping blocks of two consecutive responses into nine possible categories for each of the above sequences of behavior. Since three levers were available to the animal, considering them two at a time provided nine different categories of possible overlapping blocks $(1,1 ; 1,2 ; 1,3 ; 2,2 ; 2,3 ; 2,1 ; 3,3 ; 3,1 ; 3,2)$. Therefore, if a sample sequence of behavior (sequential lever responses) were $(1,2,3,1)$, the first and second responses would have been tabulated in the category for $(1,2)$ s, the second and third response would have been tabulated in the category for $(2,3) \mathrm{s}$, and the third and fourth responses would have been tabulated in the category for $(3,1)$ s. This procedure continued throughout the entire sequence of behavior to be analyzed.

The nine categories were then combined into three response patterns or modes, based on the direction of responding in the circular chamber. These consisted of nondifferentiated (ND) 1,$1 ; 2,2 ; 3,3$; rightgoing (R) 1,$2 ; 2,3 ; 3,1$; and left-going (L) 1,3; 3,2; 2,1 . The nondifferentiated patterns reflect responses made to the same lever. Right-going patterns reflect the direction and pattern of the reinforced chained behavior. Left-going patterns reflect the direction and pattern of behavior opposite to that of the reinforced chained behavior.

\section{Behavioral}

The mean number of total lever responses during the various phases of the experiment are presented in Table 1. Statistical analyses (t tests) showed no differences between the normal and septal groups in terms of total number of lever responses for either the 250- ot 500-reinforcement criterion groups. No differences were observed during the acquisition or extinction phases of the experiment between criterion groups on any of the measures observed. Therefore, for both the normal and septal conditions, animals of the two criterion levels are considered together in all further analyses. The failure to find differences due to the criterion level probably resulted from the fact that the animals in both criterion conditions reached an asymptotic level of performance.

A reliable difference between the normal and septal groups was found for the number of responses emitted during the acquisition phase (last $30 \mathrm{~min}$ to criterion). The septal group generated the greater number of responses $(t=2.83$, df $=16, p<.05)$ and thereby showed a higher rate of responding during this phase of the experiment. There were no
Table 1

Mean Number of Responses Produced During Each Phase of the Experiment

\begin{tabular}{ccccc}
\hline & \multicolumn{2}{c}{ To Criterion } & & \\
Group & $250 \mathrm{SR}^{\mathbf{R}}$ & $500 \mathrm{SR}$ & Acquisition & Extinction \\
\hline $\mathrm{N}$ & 2051 & 4293 & 350 & 219 \\
$\mathrm{~S}$ & 2133 & 3976 & 587 & 230 \\
\hline
\end{tabular}

Note-Acquisition refers to only the last $30 \mathrm{~min}$ of reinforced training.

$N=$ Normal

$S=$ Septal

differences between these groups with respect to the number of responses made during extinction. It should be noted that this situation occurred even though both groups had stopped responding prior to the end of the 30 -min session. Such a result seems contrary to the perseverative nature of septal lesioned rats in other extinction situations (La Vaque, 1966).

Since the septal animals made a greater number of responses during the acquisition phase, it was impossible to make comparisons to the normal animals on the basis of the frequency of $N D, L$, or $R$ responses during this portion of the experiment. However, converting these frequencies to percentages of total possible overlapping blocks of responses indicated that the two groups made an equally high proportion of reinforced right-going responses (see Table 2).

During the extinction phase of the experiment, both groups made an equal number of responses. Figure 1 shows the mean frequency of ND, L, and $\mathrm{R}$ responses made during the first extinction segment. The normal animals showed no preference for any single mode of responding during this phase of the experiment. Unlike the normal animals, however, the septal lesioned animals made a high proportion of right-going responses. At this early point in extinction, the septal animals made a reliably greater number of right-going responses than the normal animals $(\mathrm{t}=2.22, \mathrm{df}=16, \mathrm{p}<.05)$. Analyses of

Table 2

Mean Proportion of Each of the Three Response Mode Categories for Both Normal and Septal-Lesioned Groups During Acquisition* and the Two Extinction Segments

\begin{tabular}{cccc}
\hline Group & ND & L & R \\
\hline & \multicolumn{3}{c}{ Acquisition } \\
N & .156 & .072 & .772 \\
$\mathrm{~S}$ & .111 & .064 & .825 \\
& & 1st Extinction Segment & \\
$\mathrm{N}$ & .288 & .329 & .383 \\
$\mathrm{~S}$ & .139 & .334 & .527 \\
& & 2nd Extinction Segment & \\
$\mathrm{N}$ & .316 & .405 & .279 \\
$\mathrm{~S}$ & .184 & .456 & .360 \\
\hline
\end{tabular}

*Last 30 min of reinforced training $N=$ Normal $; S=$ Septal $; N D=$ Nondifferentiated $;=$ Left -going $R=$ Right -going 


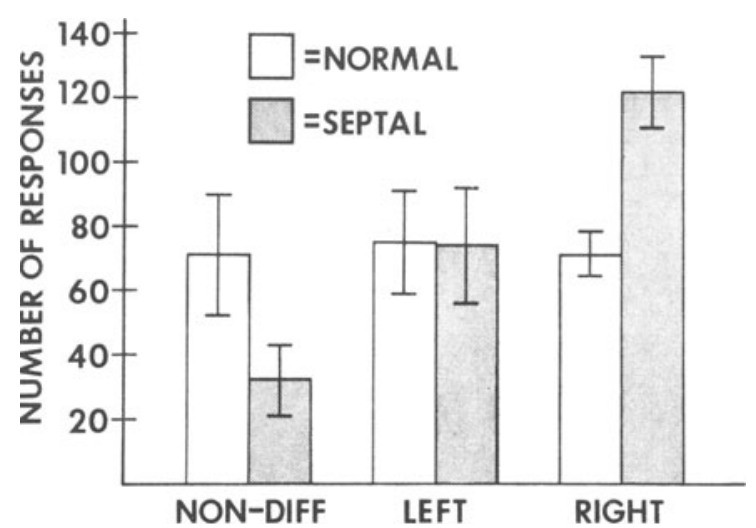

Figure 1. Mean frequency of nondifferentiated, left-going, and right-going responses emitted by the normal and septal lesioned animals during the first segment of extinction. Brackets indicate \pm 1 standard error of the mean.

the second extinction segment showed no reliable differences due to the lesion manipulation. These same results are presented as the percentage of total possible overlapping blocks of responses in Table 2, and reflect essentially the same result.

As a result of the differences in rate of responding during acquisition, correlations between the number of responses in acquisition and the frequency of right-going responses during the first extinction segment were calculated for both the normal and septal groups. In both instances, the correlations were not statistically significant, indicating that acquisition rate had no effect on extinction behavior.

\section{DISCUSSION}

In the present study, rats with septal lesions, unlike normal rats, responded in a manner which demonstrated their preferential use of response-produced cues. Although the results of this study do not give a definitive answer to the question of how septal lesioned animals use internal or external cues, it does emphasize that in certain situations rats with septal lesions can and will use response-produced cues in preference to the use of external cues. Thus, the description of the animal with septal damage as having an inability to properly use internal or response-produced cues does not seem to be adequate.

It is apparent that rats with septal lesions do differ from normal rats in their use of internal and external sets of information. Yet, despite their seeming inconsistency in cue use on various behavioral tasks, there must be an underlying definable factor controlling the septal damaged rats' behavior. One possible suggestion might center around saliency of the cue sets. Perhaps animals with septal damage require nonconflicting prominent cues to perform behavioral tasks. Subtle cues which do not stand out from the background may be useless to the septal damaged animal. Likewise, in any situation where more than one set of cues are available to allow for discrimination of the task at hand, the animal with septal damage may use the most salient of these cues. This could explain the results of the present study, where a highly stereotyped behavior is performed, providing exceedingly strong response-produced information which the septal animal uses in preference to the external cue lights provided.

Braggio and Ellen (1974) have demonstrated that by providing the septal damaged rat with differential proprioceptive feedback, differences between normal and septal rats on a DRL schedule are eliminated. It is certainly not clear, then, from Braggio and Ellen (1974), the present study, Morgan and Mitchell (1969), Sodetz (1970), and Van Hoesen et al. (1972) that the septal damaged animal even suffers from a deficiency in the use of proprioceptive information. It is apparent, contrary to some investigations (Caplan, 1973; Lubar \& Numan, 1973), that in certain situations internal response-produced information seems to be preferred and to be of clear use to animals with septal damage.

\section{REFERENCE NOTE}

1. Mitchell, J. C., \& Kratz, K. E. Facilitation of two-bar ratio performance by external cues in septal rats but not in normal rats. Paper presented at the meeting of the Society for Neuroscience, St. Louis, 1974.

\section{REFERENCES}

Braggio, J. T., \& Ellen, P. Differential proprioceptive feedback and DRL performance of normal and septal rats. Journal of Comparative and Physiological Psychology, 1974, 87, 80-90.

CaPlan, M. An analysis of the effects of septal lesions on negatively reinforced behavior. Behavioral Biology, 1973, 9, 129-167.

ELLEN, P., \& ButreR, J. External cue control of DRL performance in rats with septal lesions. Physiology and Behavior, 1969, 4, 1-6.

Ellen, P., \& Kelnhofer, M. Discrimination of response feedback following septal lesions. Psychonomic Science, 1971, 23, 94-96.

LAVAQUE, T. J. Conditioned avoidance response perseveration in septal rats during massed extinction trials. Psychonomic Science, 1966, 5, 409-410.

LUBAR, J. F., \& Numan, R. Behavioral and physiological studies of septal function and related medial cortical structures. Behavioral Biology, 1973, 8, 1-25.

Morgan, J. M., \& Mitchell, J. C. Septal lesions enhance delay of responding on a free operant avoidance schedule. Psychonomic Science, 1969, 16, 10-11.

SoDETZ, F. J. Septal ablation and free operant avoidance behavior in the rat. Physiology and Behavior, 1970, 5, 773-777.

SREBRo, B. Visual cues in successive position reversals retention deficits following septal lesions. Physiology and Behavior, 1974, 12, 639-646.

Tномаs, J. B. Stimulus perseveration and choice behavior in rats with septal lesions. Journal of Comparative and Physiological Psychology, 1972, 80, 97-105.

Van Hoesen, G. W., MacDougall, J. M., \& Mitchell, J. C. Discrimination of emitted behavior following septal area lesions in rats. Journal of Comparative and Physiological Psychology, 1972, 80, 106-122.

(Received for publication March 1976; revision received May 25, 1976.) 